\title{
Lyrodesma ET AUTRES MOLLUSQUES BIVALVES DES QUARTZITES BOTELLA (ORDOVICIEN MOYEN) DE LA ZONE CENTRE-IBÉRIQUE (ESPAGNE)
}

\author{
Juan Carlos GUTIÉRREZ-MARCO' et Claude BABIN \\ ' UEI Paleontología, Instituto de Geología Económica (CSIC-UCM), Faculdad de Ciencias \\ Geológicas, Universidad Complutense, 28040 Madrid. \\ 2 UFR Sciences de la Terre, Université Claude Bernard-Lyon I, 27-43, bd du 11 novembre, \\ 69622 Villeurbanne cédex (Francia).
}

\begin{abstract}
Gutiérrez-Marco, J.C. et Babin, C. 1999. Lyrodesma et autres mollusques bivalves des Quartzites Botella (Ordovicien Moyen) de la Zone Centre-ibérique (Espagne).[Lyrodesma and other bivalve molluscs from the Middle Ordovician Botella Quartzite (Central Iberian Zone, Spain).] Revista Española de Paleontología, $\mathbf{n}^{\circ}$ extr. Homenaje al Prof. J. Truyols, 229-238. ISSN 0213-6937.
\end{abstract}

\begin{abstract}
A rare assemblage of Middle Ordovician (upper Dobrotivian) bivalves from the Botella Quartzite Formation (upper "Tristani beds") of the Central Iberian Zone has been studied from five localities situated respectively West of Cabeza del Buey (Badajoz), South of Almadén, at Puertollano (Ciudad Real), North of Castellar de Santisteban (Jaén) and Southeast of Cabañas del Castillo (Cáceres). The assemblage is constituted by the palaeotaxodontids Praenucula costae (Sharpe), Cardiolaria beirensis (Sharpe) and Cardiolaria sp., as well as by the palaeoheterodontids Hemiprionodonta lusitanica (Sharpe), Lyrodesma truyolsi nov. sp., Modiolopsis cf. morierei de Tromelin and Modiolopsis sp. The Iberian record of Modiolopsis cf. morierei de Tromelin and the genus Lyrodesma, most probably predates its known Armorican occurrences in the upper member of the May Sandstone (Caradoc). The new species Lyrodesma truyolsi, without an anterior septum, is the oldest known representative of the genus.
\end{abstract}

Keywords: Mollusca, Bivalvia, new taxon, Ordovician, Tristani beds, Hesperian Massif, Central Iberian Zone, Spain.

\section{RÉSUMÉ}

La Formation des Quartzites Botella (Dobrotivien supérieur, Ordovicien Moyen) contient, dans ses parties inférieure et moyenne, quelques associations de bivalves qui ont été examinées dans cinq localités qui se situent à l'ouest de Cabeza del Buey (Badajoz), au sud d'Almadén et à Puertollano (Ciudad Real), au nord de Castellar de Santisteban (Jaén) et au sud-est de Cabañas del Castillo (Cáceres). Le matériel comporte les paléotaxodontes Praenucula costae (Sharpe), Cardiolaria beirensis (Sharpe) et Cardiolaria sp. et les paléohétérodontes Hemiprionodonta lusitanica (Sharpe), Lyrodesma truyolsi nov. sp., Modiolopsis cf. morierei de Tromelin et Modiolopsis sp. La présence de Modiolopsis cf. morierei de Tromelin et du genre Lyrodesma paraît précéder ici celle de ces formes dans le Massif armoricain (Grès de May supérieur, Caradoc). L'actinodontoïde Lyrodesma est décrit pour la première fois dans la Péninsule ibérique avec l'espèce L. truyolsi nov. sp., caractérisée notamment par l'absence de septum antérieur, qui semble être la forme la plus ancienne connue du genre.

Mots-clés: Mollusca, Bivalvia, nouveau taxon, Ordovicien, couches à Tristani, Massif hespérique, Zone Centre-ibérique, Espagne.

\section{RESUMEN}

La Formación Cuarcitas Botella (Dobrotiviense superior, Ordovícico Medio) contiene, en su parte inferior y media, raras asociaciones de bivalvos que han sido examinadas en cinco localidades centroibéricas. Estas se sitúan al oeste de Cabeza del Buey (Badajoz), al sur de Almadén y en Puertollano (Ciudad Real), norte de Castellar de Santisteban (Jaén) y sureste de Cabañas del Castillo (Cáceres). El material comprende los 
paleotaxodontos Praenucula costae (Sharpe), Cardiolaria beirensis (Sharpe) y Cardiolaria sp. y los paleoheterodontos Hemiprionodonta lusitanica (Sharpe), Lyrodesma truyolsi nov. sp., Modiolopsis cf. morierei de Tromelin y Modiolopsis sp. El registro de Modiolopsis cf. morierei de Tromelin y del género Lyrodesma precede muy probablemente a la aparición de estos taxones en el Macizo Armoricano (Arenisca de May superior: Caradoc). El actinodontoide Lyrodesma se describe por vez primera en la Península Ibérica, representado por la nueva especie Lyrodesma truyolsi nov. sp., carente de septo anterior, que representa la forma más antigua conocida del género.

\section{Palabras clave: Mollusca, Bivalvia, nuevo taxón, Ordovícico, Capas con Tristani, Macizo Hespérico, Zona Centroibérica, España.}

\section{INTRODUCTION}

L'étude des bivalves ordoviciens du Massif hespérique fut initiée au siècle dernier (Sharpe, 1853; de Verneuil et Barrande, 1855) puis a été reprise récemment (Babin, 1977; Young, 1985; Babin et Gutiérrez-Marco, 1985, 1991; Gutiérrez-Marco et al., 1996, 1997) dans des travaux qui ont montré la présence d'une vingtaine d'espèces de paléotaxodontes, paléohétérodontes, hétéroconches, ptériomorphes et isofilibranches, provenant pour la plupart d'entre eux des unités pélitiques centreibériques dites "couches à Tristani”. Quelques-unes de ces formes, et d'autres moins communes, se trouvent également représentées dans les faciès arénacés de la partie supérieure du Grès armoricain (Gutiérrez-Marco et cl., 1997) et dans les tempestites du Dobrotivien inférieur (Grès de los Rasos et leurs équivalents, Babin et GutiérrezMarco, 1991). Cependant, les mentions de bivalves dans les Quartzites Botella du Dobrotivien supérieur centreibérique, qui se limitaient jusqu'alors à deux localités citées par Babin et Gutiérrez-Marco (1991), se trouvent accrues par les nouvelles récoltes.

\section{CADRE GÉOLOGIQUE DES GISEMENTS ÉTUDIÉS}

La Formation des Quartzites Botella, définie dans la Sierra Morena orientale, représente l'unité quartzitique la plus commune de l'Ordovicien Moyen centre-ibérique en prenant localement d'autres dénominations de même signification stratigraphique (San José et al., 1992) telles que Quartzite Canteras (Almadén), Quartzite La Cierva (Las Villuercas), Grès de Retuerta (Monts de Tolède) et Quartzites du Torrico (Sierra de San Pedro). L'unité comprend un membre inférieur d'alternances schistogréseuses (25-150 m) et un membre supérieur de quartzites massifs (30-40 m) qui engendre un important ressaut morphologique dans toute la région (le deuxième en importance après celui généré par le Grès armoricain).

Du point de vue stratigraphique, les Quartzites Botella représentent l'unité terminale des "couches à Tristani” et leur âge a été traditionnellement attribué à la limite Llandeilo-Caradoc en l'absence généralisée de fossiles déterminants dans son membre supérieur de quartzites massifs. Cependant au cours des dernières années, ont été découvertes diverses localités géographiquement éloignées les unes des autres (Alía, Cabeza del Buey,
Aldeaquemada) où Neseuretus et d'autres fossiles du Dobrotivien supérieur persistent jusque dans la base même de l'unité pélitique sus-jacente aux quartzites. Cela implique que les Quartzites Botella appartiennent entièrement au Dobrotivien.

Du point de vue paléontologique, le membre inférieur de la formation et la base des quartzites massifs supérieurs ont fourni dans quelques rares gisements une faune très particulière de trilobites homalonotides et dalmanitides (Hammann et Henry, 1978; Hammann, 1983; Rábano, 1989a-c), brachiopodes (Mélou, 1975), conodontes (Sarmiento et al., 1995) et de rares graptolites et mollusques. L'ensemble de ces éléments indique un âge dobrotivien et, plus précisément, dobrotivien supérieur si l'on tient compte de leurs relations stratigraphiques avec des associations antérieures, recueillies au toit des Schistes Guindo sous-jacents qui correspondent au Dobrotivien inférieur terminal ou à la base du Dobrotivien supérieur suivant les localités. La partie supérieure de l'unité contient, pour sa part, une association caractéristique d'ichnofossiles ("Cruziana" almadenensis Seilacher, Rusophycus, Skolithos et autres) dépourvue de valeur biochronologique.

Ces considérations biostratigraphiques sont nécessaires pour dater, au moins de façon indirecte, les associations de bivalves des Quartzites Botella et plus particulièrement le nouveau taxon décrit ci-après. Cependant les rapports du Dobrotivien supérieur avec les séries-types de l'Ordovicien britannique ne peuvent être encore précisés en l'absence générale d'éléments de corrélation; cela est l'une des raisons qui militent en faveur de l'utilisation d'une échelle chronostratigraphique régionale pour l'Ordovicien nord-gondwanien (Gutiérrez-Marco et al., 1995). En meilleure approximation, le Dobrotivien supérieur pourrait être l'équivalent du laps de temps Llandeilien terminal-Aurelucien basal et cela ne permet pas d'écarter que les Quartzites Botella incluraient la limite Llanvirn-Caradoc. La présence non reconnue et improbable du graptolite Nemagraptus gracilis (Hall) dans ces successions méditerranéennes d'eaux peu profondes, rend impossible la datation exacte des derniers représentants du sous-genre Neseuretus, trouvés au-dessus des Quartzites Botella, partie qui reste ainsi incluse dans les "couches à Tristani" de l'Ordovicien Moyen ibérique.

Les bivalves qui font l'objet de la présente étude proviennent de cinq localités distinctes de la région centre-ibérique. Tout le matériel a été déposé au Museo Geominero (ITGE, Madrid). 
Le premier point fossilifère pour les échantillons étudiés est noté "Sierra de la Cárcel", il correspond à du matériel originel du travail de Prado (1855) conservé en grande partie au Museo Geominero (Rábano, 1998). En accord avec les données fournies par cet auteur, il s'agit de grès fossilifères trouvés près de l'entrée de la galerie du Chorrillo (flanc sud du synclinal d'Almadén) et correspondant à la partie inférieure des Quartzites Botella (= Quartzite Canteras de la Sierra de la Cárcel).

Ce matériel ancien (MGM-628-0) récolté par Prado, qui correspond parfois à une véritable lumachelle décalcifiée constituée surtout de bivalves (Pl. I, fig. 3), permet de reconnaître plusieurs des formes classiques du Dobrotivien ibérique (Babin et Gutiérrez-Marco, 1991): nombreuses Praenucula costae (Sharpe, 1853), Cardiolaria beirensis (Sharpe, 1853), Hemiprionodonta lusitanica (Sharpe, 1853) qu'accompagne sur la plaquette photographiée un exemplaire d'un rostroconche également classique à cette période, Ribeiria pholadiformis Sharpe, 1853.

La localité suivante se situe dans le prolongement oriental du flanc du même synclinal sur la commune de Puertollano (Ciudad Real). Le point se trouve au haut de la colline du Puntal, à $600 \mathrm{~m}$ à l'est du petit village d'El Villar (feuille 836, UTM: 30S-VH 412,70/4277,65) et il correspond à une intercalation de grès fossilifères découverte à la base de la principale crête quartzitique du membre supérieur des Quartzites Botella (localement reconnaissable par son relief et par l'abondance de Daedalus isp. de grand diamètre). En plus de rares restes de trilobites (Iberocoryphe sp.) et de gastéropodes, ce gisement a livré quelques Cardiolaria sp., Hemiprionodonta sp., Modiolopsis sp. et M. cf. morierei, mais il est surtout intéressant par la présence de lyrodesmes que nous attribuons à une nouvelle espèce, Lyrodesma truyolsi.

A l'est d'Almadén, la région de Cabeza del Buey (Badajoz, flanc nord du synforme de Los Pedroches) présente d'intéressants affleurements des Quartzites Botella dans lesquels Prost-Dame (1980) découvrit quelques horizons fossilifères. Le point 9 de cet auteur se situe juste à la Casa de Artobás, immédiatement à l'est du $\mathrm{km} 59$ de la route $\mathrm{N}-420$ (feuille 806, UTM: 30S-TH 297,65/4284,40). Les niveaux de grès correspondant au tiers inférieur des Quartzites Botella y ont livré divers trilobites [Iberocoryphe verneuili Hammann, Eohomalonotus sp., Plaesiocomia oehlerti (Kerforne), Crozonaspis incerta (Deslongchamps)], des restes indéterminables de brachiopodes articulés, de gastéropodes et d'ostracodes et une plaquette portant quatre moules internes et une quinzaine de moules externes de bivalves que nous désignons Modiolopsis sp.

Dans la région d'Aldeaquemada (Sierra Morena orientale), les premiers 10-20 mètres de quartzites en gros bancs appartenant aux Quartzites Botella ont fourni plusieurs gisements fossilifères comme celui situé sur la commune de Castellar de Santisteban (Jaén), à 2,5 km au nord-est de la maison de La Alameda (feuille 863, UTM: 30S-VH 482,10/4253,10). Ce point, qui fut découvert par Carré et al. (1971), contient de nombreux trilobites,

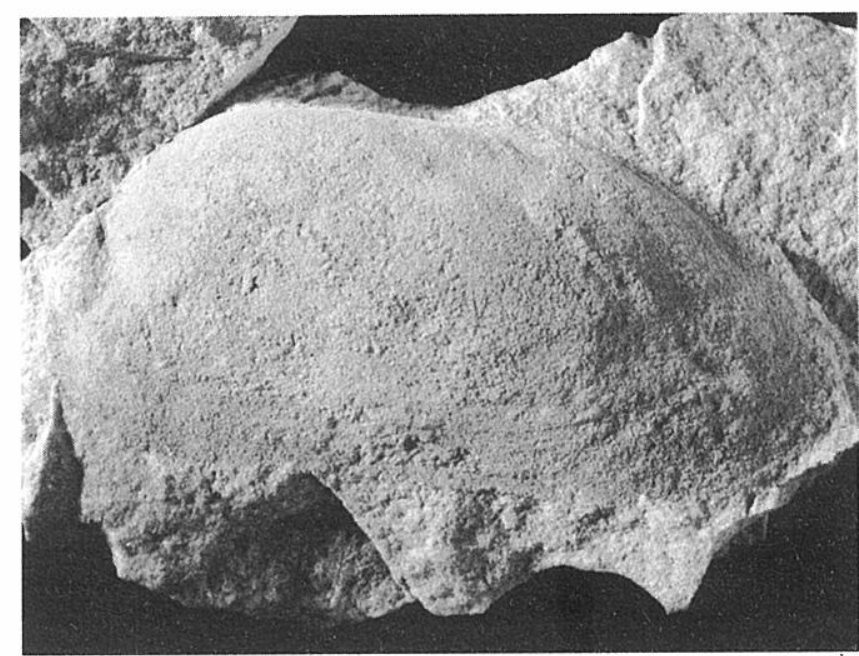

Figure 1. Modiolopsis cf. morierei de Tromelin, 1877. Moule interne de valve gauche, Quartzites Botella, Dobrotivien supérieur, Puntal del Villar (Ciudad Real). MGM-761-O. x 1,5.

brachiopodes et de rares mollusques; Mélou (1975), Hammann et Henry (1978), Hammann (1983), Rábano (1989a-c) et Babin et Gutiérrez-Marco (1991) y ont identifié Eohomalonotus brongniarti (Deslongchamps), E. vicaryi (Salter), E. sdzuyi Hammann et Henry, Iberocoryphe verneuili Hammann, Iberocoryphe $\mathrm{n}$. sp. aff. fugitiva (de Tromelin), Kerfornella brevicaudata (Deslongchamps), Plaesiacomia oehlerti (Deslongchamps), Neseuretus henkei Hammann, Crozonaspis incerta (Deslongchamps), C. armata Hammann, Heterorthina kerfornei Mélou, Eorhipidomella cf. musculosa (Mélou) et Cardiolaria beirensis (Sharpe). Le nouveau matériel n'est fait que de mauvais moules dont trois sont probablement des Cardiolaria beirensis tandis qu'un moule interne partiel et un moule externe de plus grandes dimensions, respectivement de 35 et $40 \mathrm{~mm}$ de longueur, restent indéterminables.

Enfin, nous pouvons désormais préciser la localisation du gisement de "La Víbora" pour lequel nous avions signalé précédemment (Babin et Gutiérrez-Marco, 1991) la présence de grès très fossilifères avec Praenucula costae (Sharpe), Cardiolaria beirensis (Sharpe) et Glyptarca? lusitanica (Sharpe). La provenance exacte de ce matériel, trouvé en 1973 par Ph. Rossi, n'avait pu être établie alors avec exactitude et fut considérée comme correspondant probablement au Quartzite du Torrico (= Botella) dans un lieu homonyme (La Víbora) de la Sierra de San Pedro, à $7 \mathrm{~km}$ au sud d'Aliseda (Cáceres). En réalité, un courrier retrouvé de $\mathrm{Ph}$. Rossi, datant du 24 avril 1974, permet de localiser cet affleurement dans la moitié inférieure des Quartzites Botella sur le flanc sudouest du synclinal de Santa Lucía (région de Las Villuercas). L'arrêt, dit "La Mata de La Víbora" (feuille 707, UTM approx. 30S-TJ 290,80/4370,05) situé sur la commune de Cabañas del Castillo (Cáceres) à l'ouest du $\mathrm{km} 9$ de la route de Navezuelas à Cañamero, constitue le seul affleurement avec des bivalves identifiables connu dans les Quartzites Botella (= Quartzite de La Cierva) de 
la région de Las Villuercas. Comme dans la Sierra de la Cárcel (Almadén), les bivalves sont ici associés au rostroconche Ribeiria pholadiformis Sharpe, particulièrement abondant dans cet affleurement.

\section{SYSTÉMATIQUE}

L'état de conservation du matériel, sous forme de moules internes et externes, est souvent assez fruste et certaines déterminations demeurent donc imprécises. Quelques-unes des espèces signalées méritent néanmoins de brèves remarques.

\section{CLASSE BIVALVIA Linnaeus, 1758 SOUS-CLASSE PALAEOHETERODONTA Newell, 1965 \\ ORDRE ACTINODONTOIDA Douvillé, 1912 \\ Superfamille GLYPTARCOIDEA Cope, 1996 \\ Famille Glyptarcidae Cope, 1996 \\ Genre Hemiprionodonta Cope, 1996}

Espèce-type: Dolabra? lusitanica Sharpe, 1853, de l'Ordovicien moyen du Portugal.

\section{Hemiprionodonta lusitanica (Sharpe, 1853)}

v* 1853 Dolabra? lusitanica Sharpe, 151, pl. 9, fig. 3.

v. 1991 Glyptarca? lusitanica (Sharpe); Babin et Gutiérrez-Marco, 126, text-fig. 6a-f.

\section{Observations}

Cette forme, qui est très commune dans l'Ordovicien Moyen de la Péninsule ibérique et du Massif armoricain, a été assignée à différents genres (voir synonymie in Babin et Gutiérrez-Marco, 1991). Nous avions proposé, dans la première forme du manuscrit soumis pour notre publication de 1991, la création d'un nouveau genre afin de souligner l'originalité, selon nous apparente, de cette espèce. L'un des rapporteurs anonymes jugeant cette proposition non justifiée, préféra son maintien dans le genre Glyptarca Hicks, 1873 comme l'avait fait Morris (1979); afin de marquer notre réserve, nous la désignâmes Glyptarca? lusitanica. C'est donc avec satisfaction que nous avons constaté que Cope (1996), dans son importante étude des bivalves arenigiens du Pays de Galles, attribue, à l'issue d'une redéfinition précise de Glyptarca, l'espèce ibéro-armoricaine à un nouveau genre qu'il nomme Hemiprionodonta (Cope, 1996, p. 991) et place dans une nouvelle famille Glyptarcidae. C'est cette nomenclature que nous adoptons ici.

\section{ORDRE TRIGONIOIDA Dall, 1889 Superfamille TRIGONIOIDEA Lamarck, 1819 \\ Famille Lyrodesmatidae Ulrich, 1894 \\ Genre Lyrodesma Conrad, 1841}

Espèce-type: Lyrodesma planum Conrad, 1841.

\section{Observations}

A l'occasion de cette première citation de Lyrodesma dans la péninsule Ibérique, il est opportun de faire le point sur notre connaissance actuelle au sujet de ce genre. Un premier historique rapide le concernant fut donné par Babin (1966, p. 237) pour en souligner l'originalité, rejeter le rapprochement effectué par les anciens auteurs avec les paléotaxodontes et adopter la proposition de Cox (1960) d'un placement parmi les pantodontides qui regroupaient Actinodontidae Douvillé (= Cycloconchidae Ulrich), Lyrodesmatidae Ulrich et Redoniidae Babin. Ce point de vue est désormais celui adopté par la majorité des auteurs qui placent ces différentes familles dans la sous-classe Palaeoheterodonta créée par Newell (1965) ou dans celle des Heteroconchia Hertwig (Pojeta, 1987).

Depuis, divers éléments sont venus enrichir la connaissance de la famille et du genre. Pojeta (1971, pl. 3) a donné de belles illustrations des espèces nordaméricaines dont il convient de rappeler qu'elles proviennent toutes de l'Ordovicien Supérieur de l'échelle stratigraphique standard. Il suggérait que le genre Lyrodesma éclaterait probablement en plusieurs genres s'il était mieux connu. L'auteur figurait également (Pojeta, 1971, pl. 3, fig. 11 et 23) deux formes armoricaines, L. armoricana de Tromelin et Lebesconte, 1875 et $L$. caelata Salter, 1864 . Pour la première de ces deux espèces, décrite dans les Grès armoricains supérieurs (Arenig) de Bretagne, Pojeta et GilbertTomlinson (1977, p. 30) proposèrent, lors d'une étude des bivalves de l'Ordovicien d'Australie, de la placer avec réserve dans un genre alors créé, en la nommant Noradonta? armoricana. Cependant Babin (1982) justifiait la création d'un nouveau taxon, Tromelinodonta, pour cette espèce. Pojeta (1971, p. 11, fig. 1 D, E),

\section{Planche I}

1, 2, 4, 5 Lyrodesma truyolsi nov. sp. Quartzites Botella, Dobrotivien supérieur, Puntal del Villar. 1. Holotype, moule interne d'une valve gauche. MGM-749-O. x 3. 2. Paratype, détail de la denture montrant les faces microcrénelées des dents, agrandissement de l'exemplaire de la fig. 4. MGM755-O. x 9. 4. Paratype, moule interne très partiel d'une valve droite montrant la denture. MGM-755O. x 3. 5. Paratype, moule interne d'une valve droite exposant la denture. MGM-756-O b. x 3.

Plaquette des Quartzites Botella de la Sierra de la Cárcel (Almadén, Ciudad Real), coll. Prado (MGM-628-O) portant notamment de nombreux moules internes et externes de bivalves [Cardiolaria beirensis (Sharpe), Praenucula costae (Sharpe), Hemiprionodonta lusitanica (Sharpe)]. x 1,5. 


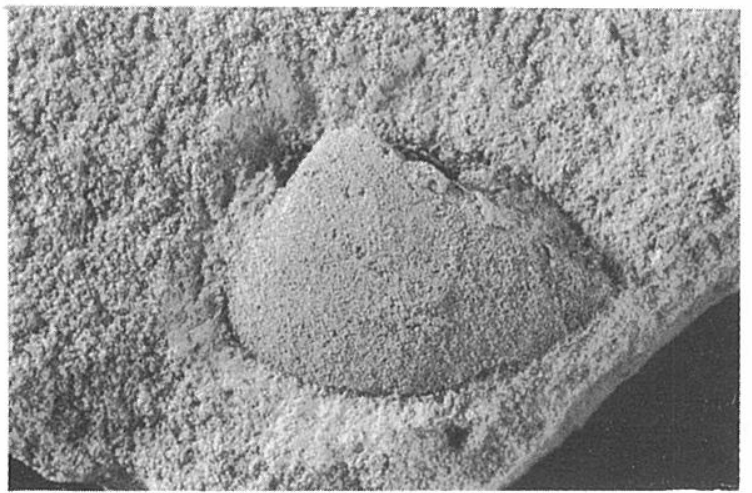

Planche I

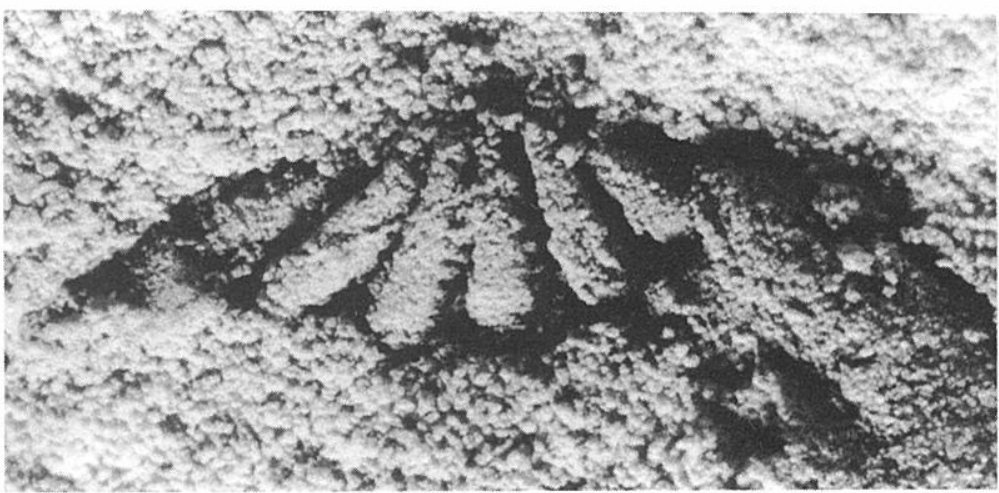

1

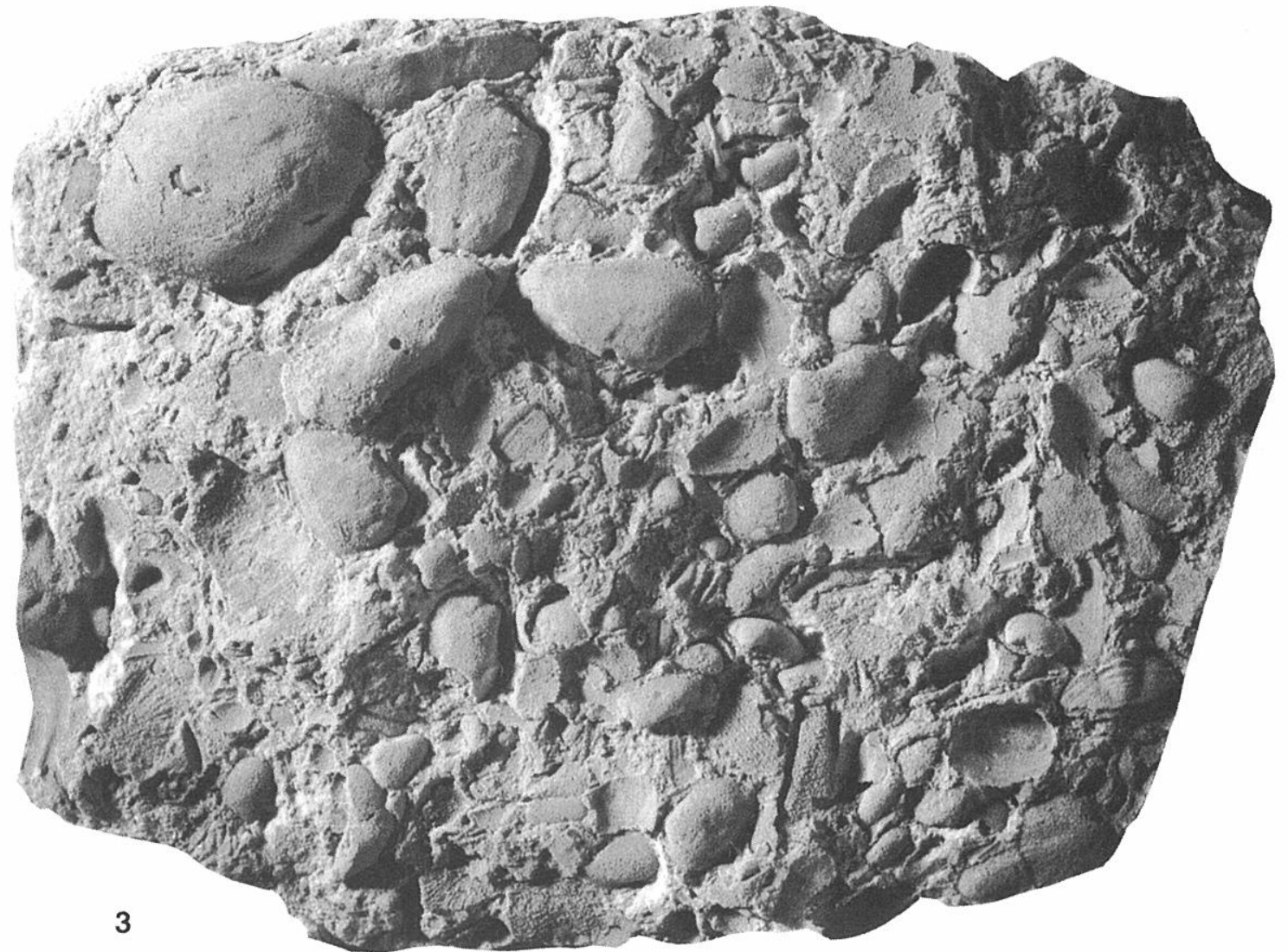

4
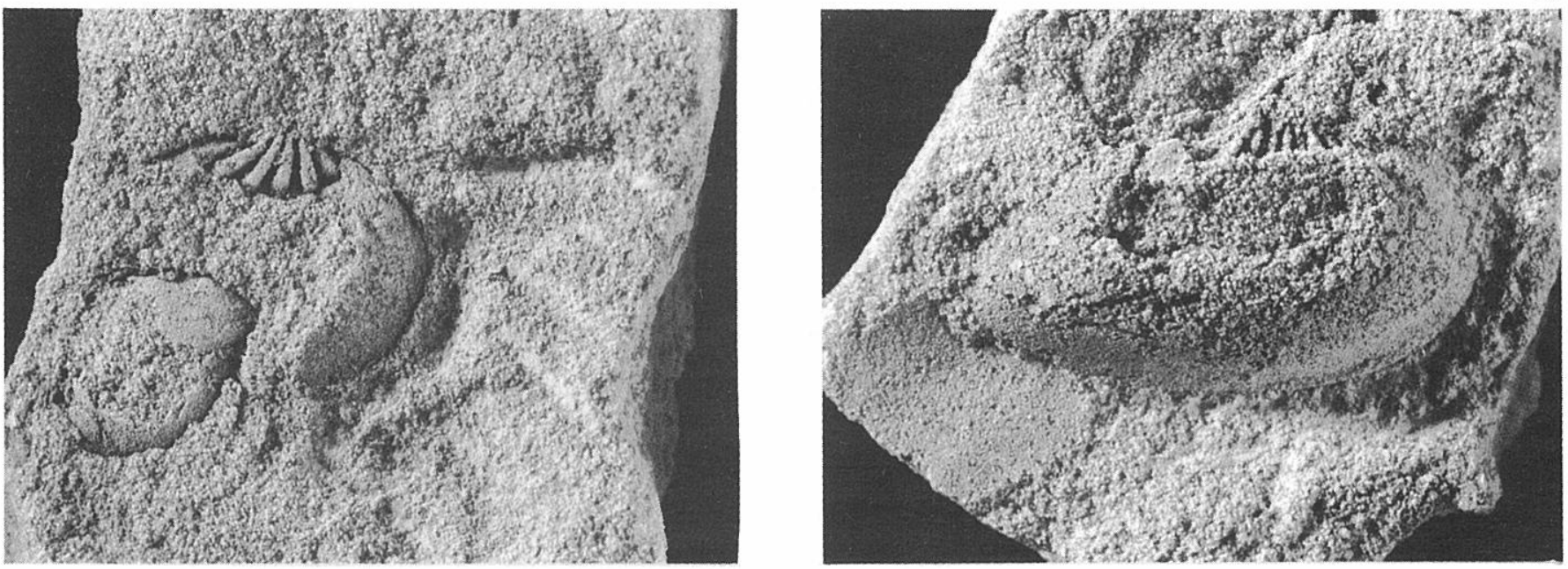

Revista Española de Paleontología, nº extr. Homenaje al Prof. J. Truyols, 1999. 
reproduisant les figures originales de l'auteur attribua au genre Lyrodesma un autre bivalve décrit par Barrois (1891, p. 192, pl. 1, fig. 9a, b) dans le Grès armoricain sous la désignation Nuculites acuminata Barrois, adoptée sans réserves durant plus d'un demi-siècle (Péneau, 1946, p. 63 , pl. 2, fig. 28). Si l'on doit convenir avec Pojeta (1971) que la forme de ces valves peut aisément s'intégrer dans la morphologie des lyrodesmes, il convient pourtant de rappeler que ce matériel, conservé au Musée de Nantes et autrefois réexaminé par l'un de nous (Babin, 1966, p. 95, pl. 3, fig. 3), est pauvre, extrêmement fruste, ne montre aucun caractère de la charnière et qu'il ne peut pas être attribué génériquement avec certitude.

Par ailleurs, Pojeta et Gilbert-Tomlinson (1977) ayant introduit un autre genre Brachilyrodesma pour l'Ordovicien australien, la famille Lyrodesmatidae se trouve comporter actuellement quatre genres. La répartition géographique des lyrodesmatides a été récemment étendue à l'Amérique du Sud avec la découverte de Lyrodesma sp. dans l'Ordovicien supérieur de Bolivie (Sánchez et Suárez-Soruco 1996).

Tandis que des clichés classiques de Lyrodesma, souvent des mêmes exemplaires adultes, se retrouvaient dans diverses publications concernant les bivalves ordoviciens (Pojeta, 1978, 1987; Pojeta et Runnegar, 1985), une intéressante documentation était fournie par Harrison et Harrison (1975). Ces auteurs ont décrit et figuré des individus juvéniles (p. 215; pl. 2, fig. 14-17) d'un Lyrodesma sp. dans des faunules de l'Ohio rapportées au Silurien inférieur. Cette présence qui étend l'extension stratigraphique du genre confirme ainsi celle déjà notée dans le Llandovery de Grande-Bretagne (Ziegler et al., 1968). L'examen des dentures de formes juvéniles constitue, en outre, un intéressant élément pour les relations phylogénétiques comme l'a remarqué Waller (1990, p. 65).

\section{Lyrodesma truyolsi nov. sp. Pl. I, figs. 1, 2, 4, 5}

Holotype: MGM-749-O, moule interne d'une valve gauche (Pl. I, fig 1).

Paratypes: MGM-750-O, 751-O, 752-O, 753-O, 754-O, 755-O, 756-O a et b, 757-O a et b.

Localité-type: $100 \mathrm{~m}$ à l'est du sommet de la colline "Puntal del Villar", immédiatement à l'est du village de El Villar (Puertollano, Ciudad Real).

Strate-type: Quartzites Botella, $2 \mathrm{~m}$ au-dessus de la base de la barre quartzitique supérieure, Dobrotivien.

Origine du nom: en l'honneur du Prof. D. Jaime Truyols.

Matériel: Moules internes univalves souvent très partiels; six valves, trois gauches et trois droites, montrent des vestiges de denture (holotype et paratypes MGM-750-O à
MGM-754-O); cinq dentures, assez bien conservées, sont celles de trois valves droites et de deux valves gauches, toutes extrêmement partielles (paratypes MGM-755-0, MGM-756-O a et b, MGM-757-O a et b); trois autres valves (deux gauches, une droite) sans denture observable (MGM758-O à MGM-760-O) sont néanmoins attribuables avec certitude à la même espèce. Dans le reste du matériel, il est probable que quelques moules, partiels et dépourvus de dents, sont aussi des lyrodesmes.

\section{Diagnose}

Lyrodesma dépourvu de septum antérieur et sans empreintes adductrices nettement circonscrites.

Lyrodesma without anterior septum and without clearly defined adductor scars.

\section{Description}

La coquille, dont la longueur varie de 10 à $20 \mathrm{~mm}$ dans le matériel disponible, est assez peu convexe et offre un contour subquadrangulaire avec un crochet, bien marqué, situé vers le tiers antérieur. En arrière de celui-ci la ligne cardinale est rectiligne avant de se raccorder au côté postérieur obliquement tronqué. Les bords ventral et antérieur sont régulièrement convexes. Une carène mousse qui s'étend de l'umbo au côté postérieur détermine un talus dorso-postérieur faiblement déprimé. La denture comporte 6 à 9 dents cardinales fortement microcrénelées sur leurs deux faces et disposées, suivant l'habitus caractéristique du genre, en un éventail convergeant vers le crochet. Les autres caractères internes ne sont généralement pas observables. Sur quelques rares exemplaires, un très vague relief suggère la présence d'une empreinte adductrice postérieure sur le talus dorsal. Seule une valve droite (MGM-751-O) porte un petit sillon, peut-être accentué par déformation (?), qui correspondrait à un septum placé devant le muscle adducteur postérieur.

Cette espèce de Lyrodesma n'est pas aisément assimilable à l'une des espèces antérieurement décrites dans le Massif armoricain. L. securis de Tromelin et Lebesconte, 1875 (= L. dufeti de Tromelin et Lebesconte, 1875; $L$. gallica Munier-Chalmas, 1876 et L. sacheri MunierChalmas, 1876) présente une empreinte adductrice antérieure bien marquée et limitée par un septum postérieur; il en est de même pour $L$. caelata Salter, 1864 qui porte, en outre, un septum devant l'adducteur postérieur. L. lebesconti Munier-Chalmas, 1876, démunie de septum antérieur, montre, en revanche sur les moules internes, une assez forte incisure qui correspond à un septum en avant de l'empreinte adductrice postérieure.

Il paraît donc justifié, malgré le caractère un peu sommaire du matériel, de distinguer l'espèce ibérique de celles antérieurement décrites. L'holotype a été choisi pour disposer d'une valve presque entière portant simultanément une partie de sa charnière; l'ensemble des paratypes désignés, qui comprend des charnières isolées, permet d'obtenir une image plus complète des caractéristiques de l'espèce. 
ORDRE MODIOMORPHOIDA Newell, 1969 Superfamille MODIOMORPHOIDEA Miller, 1877 Famille Modiolopsidae Fischer, 1887

Genre Modiolopsis Hall, 1847

Espèce-type: Pterinea modiolaris Conrad, 1838 par désignation originale (Hall, 1847, p. 157).

\section{Modiolopsis cf. morierei de Tromelin, 1877} Fig. 1

cf. 1877 Modiolopsis Morierei de Tromelin, 40.

cf. 1890 Modiolopsis Morieri (sic) de Tromelin; Bigot, 795, pl. 22, fig. 4.

cf. 1966 Modiolopsis morierei de Tromelin; Babin, 188, pl. 7, fig. 8.

Matériel: Quatre moules internes de valves gauches, plus ou moins complets, l'un accompagné de son moule externe, provenant tous de Puntal del Villar, sont des Modiolopsis d'assez grandes dimensions (longueurs respectives d'environ 42, 46, 52 et 55 mm). MGM-761-O à MGM-764$\mathrm{O}$ inclus.

\section{Description}

Il s'agit de coquilles assez peu convexes avec une carène umbono-postéro-palléale mousse qui délimite un talus postéro-dorsal peu convexe et un flanc portant une très discrète dépression umbono-palléale. Le crochet, platement arrondi, est placé vers le $1 / 5^{\mathrm{e}}$ antérieur. Derrière lui, le bord cardinal est rectiligne, légèrement relevé vers l'arrière ou faiblement convexe. Le bord postérieur détermine un angle très obtus (environ $150^{\circ}$ ) avec le côté cardinal puis se raccorde par une extrémité convexe avec le côté ventral subrectiligne; le côté antérieur, court, est largement arrondi. La charnière est édentule dans sa partie postérieure, la partie umbonale n'en est pas dégageable. Les quatre moules internes portent une empreinte adductrice très antérieure, ovale et assez fortement imprimée dans la coquille. Aucune autre empreinte n'est discernable. L'ornementation est suggérée par quelques sillons concentriques sur deux des moules.

\section{Discussion}

Par son contour, cette forme est proche de certains des nombreux Modiolopsis autrefois figurés en Normandie (Salter, 1864; Wyatt Edgell, 1874; Bigot, 1890). Parmi ces "espèces" dont certaines sont peut-être artificielles par suite d'un polymorphisme non reconnu, c'est avec $M$. prima (d'Orbigny) et $M$. morierei de Tromelin, qui ont été illustrées par Bigot (1890, la seconde étant nommée morieri, p. 795) puis révisées par Babin (1966), que l'on peut comparer les fossiles des Quartzites Botella. L'allongement de la coquille plaide plus précisément en faveur d'un rapprochement avec M. morierei mais la

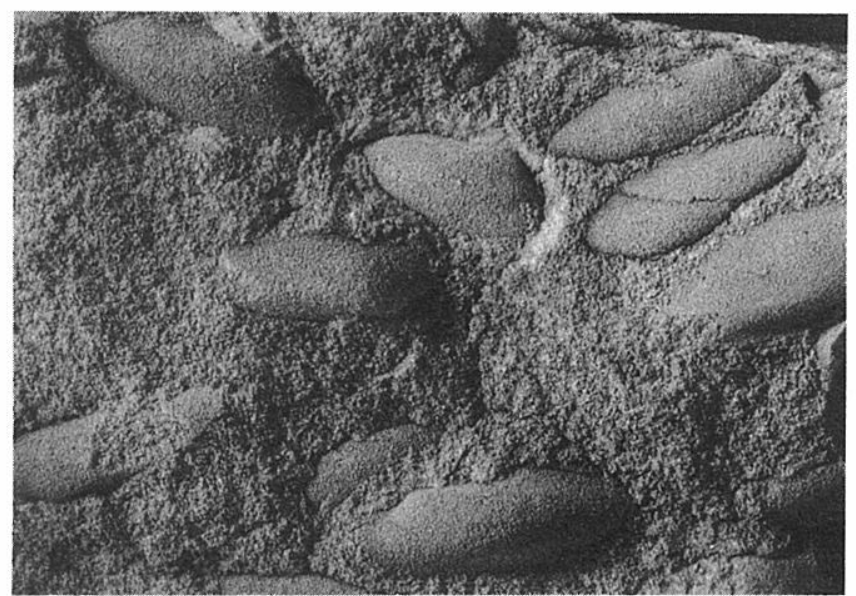

a

b

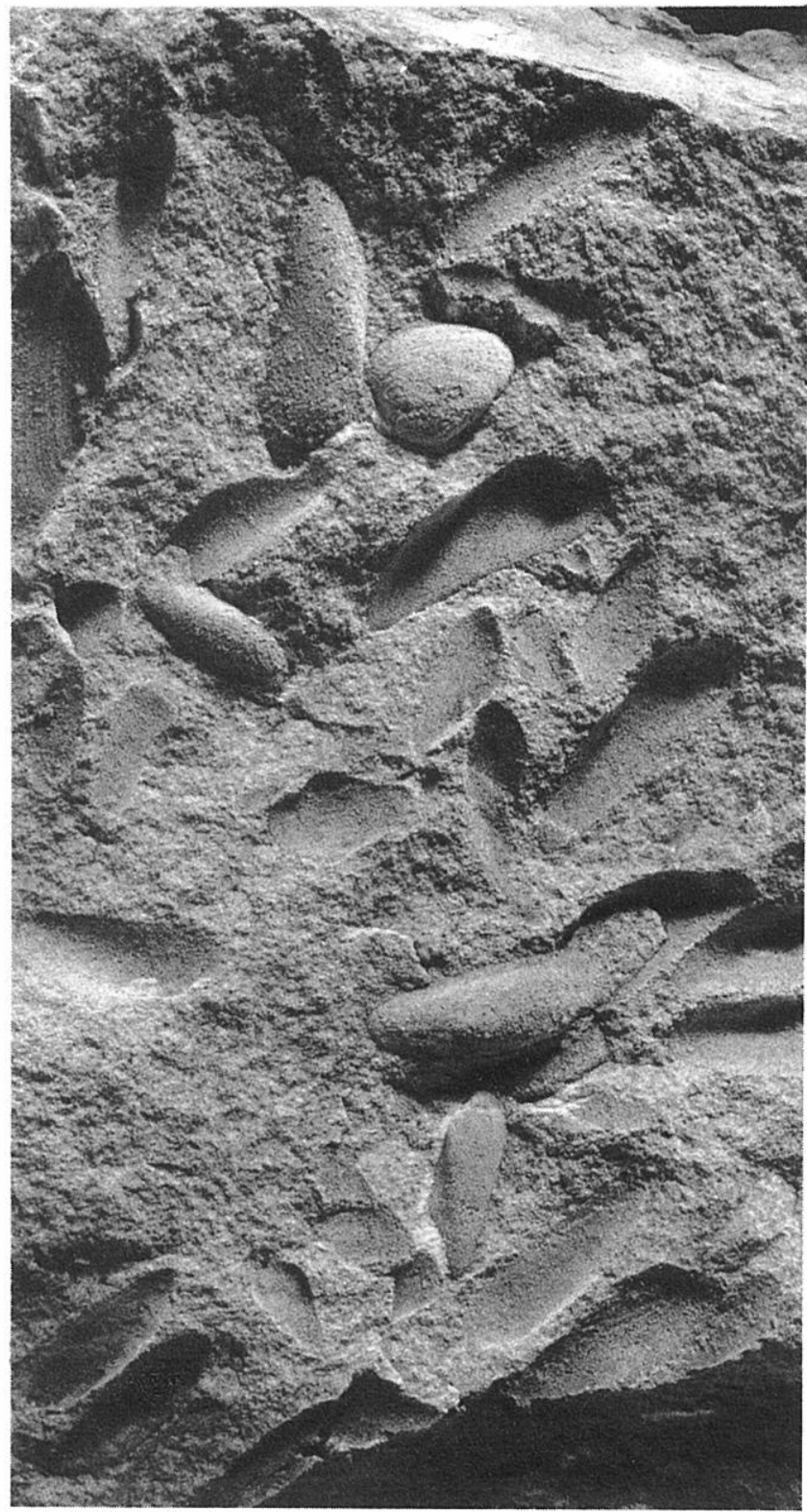

Figure 2. a. Modiolopsis normanniana (d'Orbigny, 1850). Partie d'une plaquette portant de nombreux moules internes. Grès de May, Caradoc?, May/Orne (Calvados, France). FSL 550262. x 1,5. b. Modiolopsis sp. Partie d'une plaquette portant de nombreux moules internes et externes. Quartzites Botella, Dobrotivien supérieur, Cabeza del Buey (Badajoz). MGM-765-O. x 1,5. 
convexité des exemplaires ibériques est probablement moindre que chez ceux de Normandie; de façon générale, la pauvreté des caractères diagnostiques incite à ne proposer qu'une attribution approchée.

\section{Modiolopsis sp.}

Fig. 2b

Matériel: MGM-765-O, une plaquette de Cabeza del Buey; MGM-766-O, moule interne d'une valve gauche de Puntal del Villar.

\section{Observations et discussion}

Au gisement Cabeza del Buey, de petits bivalves allongés, mesurant de 11 à $20 \mathrm{~mm}$ pour la vingtaine d'exemplaires disponibles, sont désignés Modiolopsis sp. Un moule interne de valve gauche du gisement de Puntal del Villar en est conspécifique.

En avant du crochet, placé vers le sixième antérieur de la longueur totale, la ligne cardinale est concave; en arrière de celui-ci, elle est rectiligne sur environ un tiers de la longueur de la coquille puis est oblique pour se raccorder au côté ventral par une extrémité postérieure allongée et convexe. Le côté antérieur est fortement convexe; le bord palléal est rectiligne ou présente une très faible concavité médiane. La coquille a ainsi un aspect très allongé avec des côtés cardinal et ventral subparallèles et un bord postérieur obliquement tronqué. La convexité de ces valves est faible; une carène mousse, qui s'étend du crochet à l'angle postéro-ventral, délimite un talus postéro-dorsal assez abrupt. Une très discrète dépression peut précéder la carène, il lui correspond alors une faible sinuosité du bord ventral. Les caractères internes ne sont pas observables; un moule interne paraît montrer un septum derrière le muscle adducteur antérieur mais il s'agit d'un artefact de fossilisation; la charnière semble édentule mais la partie umbonale n'est pas observable. Les moules externes ne montrent pas davantage l'ornementation, probablement concentrique, de ces coquilles.

La morphologie générale de ces petits fossiles ne permet pas d'exclure qu'il s'agisse de solemyoïdes comme Psiloconcha mais l'extrémité postérieure de ces derniers est, en général, moins acuminée. C'est une attribution au genre Modiolopsis qui est la plus plausible et que nous retenons; ce genre n'est pas rare dans l'Ordovicien moyen mais les espèces antérieurement décrites diffèrent de nos fossiles.

Une forme très voisine examinée par Bigot (1890, p. 800 , pl. 22, fig. 6) sous 1'appellation Orthonota? normanniana (d'Orbigny) est cependant connue des Grès de May en Normandie. Nous figurons de nouveau (Fig. 2a), afin de compléter l'illustration ancienne de Bigot, cette espèce d'après du matériel trouvé dans la collection de Verneuil à l'Université de Lyon I (FSL 550262). Constituant probablement, à l'instar de la forme ibérique, des populations abondantes dans certains niveaux comme le suggère la fossilisation groupée de nombreuses valves, Modiolopsis normanniana diffère de $M$. sp. par un bord ventral légèrement convexe ce qui modifie également le profil acuminé de l'extrémité postérieure. Le bivalve ibérique paraît donc être original mais l'état du matériel n'autorise guère l'établissement d'une espèce nouvelle.

\section{INTÉRET DE CES FAUNES DE BIVALVES}

D'un point de vue paléoécologique, la présence des espèces ici indiquées dans les Quartzites Botella vient confirmer l'abondance locale, à l'Ordovicien moyen, de ces bivalves, tous endobenthiques, dans les environnements internes des plates-formes continentales. Plusieurs des espèces reconnues (Hemiprionodonta lusitanica, Praenucula costae, Cardiolaria beirensis) étaient très communes dans les vases de l'Ordovicien moyen du domaine ibéro-armoricain; elles étaient, ici, les hôtes de sables fins. On peut noter, en revanche, l'absence d'autres espèces souvent très communes dans les vases et appartenant aux genres Myoplusia Neumayr, Redonia Rouault ou Eurymya Ulrich.

Les Modiolopsis ordoviciens paraissent avoir préféré souvent, pour leur part, les milieux sableux; ils sont particulièrement abondants, par exemple, dans les Grès de May en Normandie où ils atteignent de grandes dimensions; leur présence dans les Quartzites Botella confirme ces préférences granulométriques pour le substrat. En Espagne, le genre a aussi été signalé récemment dans le Grès armoricain (Gutiérrez-Marco et al., 1997).

En ce qui concerne la répartition stratigraphique comparée de ces gisements espagnols avec le Massif armoricain, le cas des Modiolopsis et des Lyrodesma pourrait rester ambigu. Tandis qu'ils sont présents ici dans des niveaux du Dobrotivien, ils ont été cités, essentiellement pour le premier et uniquement pour le second, du Caradoc dans le Massif armoricain.

Nous avons montré que l'espèce ibérique de Lyrodesma peut être distinguée de celles décrites dans le Grès de May en Normandie ou dans celui de StGermain/Ille à La Bouëxière en Bretagne. Un âge différent n'implique donc aucune équivoque.

Pour les modiolopsides, en revanche, nous avons souligné les affinités apparentes avec des formes décrites dans le Caradoc en Normandie. En réalité, l'âge des formations normandes concernées reste imprécis. Ces bivalves sont cités par Bigot (1890) des Grès de May sans autre précision; nous savons désormais que les "grès de May inférieurs" sont dobrotiviens. L'une des localités souvent rapportée par Bigot est Besneville. Or, la présence dans la Formation de Besneville, du trilobite Homalonotus bonissenti Morière que Henry (1980, p. 101) a revu en l'attribuant au genre Iberocoryphe apporte, selon cet auteur, peu de précisions chronologiques mais, par analogie avec des données ibériques, Henry serait enclin à considérer l'espèce comme llandeilienne. Pour sa part, Robardet (1981) hésite dans ses colonnes stratigraphiques, pour la Formation du Mont de Besneville, entre un âge entièrement llandeilien (Robardet, 1981, p. 27) et un âge 
partiellement caradocien pour la partie sommitale (Robardet, 1981, p. 80, 83).

Pour ce qui est de la paléobiogéographie enfin, l'apport nouveau est fourni par l'extension à la péninsule ibérique de la présence du genre Lyrodesma dont cela semble constituer simultanément la plus ancienne occurrence connue puisqu'elle est probablement llandeilienne.

\section{REMERCIEMENTS}

Le travail de terrain de l'un de nous (JC G-M) a été financé par le Project PB96-0839 de la DGES. Les photographies ont été réalisées par Noël Podevigne (Lyon). Isabel Rábano (Museo Geominero, Madrid) a déterminé les trilobites de Cabeza del Buey et d'El Villar et a facilité le dépôt du matériel au musée; Ph. Rossi nous a autrefois confié le matériel qu'il collecta à "La Víbora".

Les commentaires des deux rapporteurs, M.T. Sánchez et E.L. Yochelson nous ont permis d'améliorer le manuscrit initial.

Cette note constitue une contribution aux projets PICG 410 "La grande biodiversification ordovicienne" et DGES PB96-O839 (Ministerio de Educación y Cultura de España).

\section{RÉFÉRENCES}

Babin, C. 1966. Mollusques bivalves et céphalopodes du Paléozoïque armoricain. Etude systématique. Essai sur la phylogénie des Bivalves. Esquisse paléoécologique. Imprimerie Commerciale et Administrative, Brest, 1471.

Babin, C. 1977. Étude comparée des genres Babinka Barrande et Coxiconcha Babin (Mollusques Bivalves de l'Ordovicien). Intérêt phylogénétique. Geobios, 10, 51-59.

Babin, C. 1982. Tromelinodonta nov. gen., bivalve lyrodesmatide (Mollusca) de l'Arenigien (Ordovicien inférieur). Geobios, 15, 423-427.

Babin, C. et Gutiérrez-Marco, J.C. 1985. Un nouveau cycloconchide (Mollusca, Bivalvia) du Llanvirn inférieur (Ordovicien) des Monts de Tolède (Espagne). Geobios, 18, 609-616.

Babin, C. and Gutiérrez-Marco, J.C. 1991. Middle Ordovician bivalves from Spain and their phyletic and palaeogeographic significance. Palaeontology, 34, 109147.

Barrois, C. 1891. Mémoire sur la faune du Grès armoricain. Annales de la Société géologique du Nord, 19, 134-237.

Bigot, A. 1890. Note sur quelques Pélécypodes des grès siluriens de l'Ouest de la France. Bulletin de la Société géologique de France, [3], 17, 791-801.

Carré, D., Henry, J.-L., Poupon, G. et Tamain, G. 1971. Les quartzites Botella et leur faune trilobitique. Le problème de la limite Llandeilien-Caradocien en Sierra Morena. Bulletin de la Société géologique de France, [7], 12, 774-785.

Conrad, T.A. 1841. On the palaeontology of the state of New York. New York State Geological Survey, 5th
Annual Report, 25-27.

Cope, J.W. 1996. Early Ordovician (Arenig) bivalves from the Llangynog Inlier, South Wales. Palaeontology, 39, 979-1025.

Cox, L.R. 1960. Thoughts on the classification of the Bivalvia. Proceedings of the Malacological Society of London, 34, 60-88.

Gutiérrez-Marco, J.C., Rábano, I., San José, M.A., Herranz, P. and Sarmiento, G.N. 1995. Oretanian and Dobrotivian stages vs. "Llanvirn-Landeilo" Series in the Ordovician of the Iberian Peninsula. In Ordovician Odyssey (Eds. J.D. Cooper, M.L. Droser and S.C. Finney). Pacific Section Society for Sedimentary Geology, Book 77, 5559.

Gutiérrez-Marco, J.C., Albani, R., Aramburu, C., Arbizu, M., Babin, C., García-Ramos, J.C., Méndez-Bedia, I., Rábano, I., Truyols, J., Vannier, J. y Villas, E. 1996. Bioestratigrafía de la Formación Pizarras del Sueve (Ordovícico Medio) en el sector septentrional de la Escama de Laviana-Sueve (Zona Cantábrica, $\mathrm{N}$ de España). Revista Española de Paleontología, 11, 48-74.

Gutiérrez-Marco, J.C., Babin, C. y Porro Mayo, T. 1997. Moluscos bivalvos de las facies cuarcíticas del Ordovícico Inferior centroibérico. Geogaceta, 22, 85-88.

Hall, J. 1847. Palaeontology. Geological Survey of New York, 1, XXIII + 1-338.

Hammann, W. 1983. Calymenacea (Trilobita) aus dem Ordovizium von Spanien; ihre Biostratigraphie, Ökologie und Systematik. Abhandlungen der Senckenbergischen naturforschenden Gesellschaft, 542, 1-177.

Hammann, W. et Henry, J.-L. 1978. Quelques espèces de Calymenella, Eohomalonotus et Kerfornella (Trilobita, Ptychopariida) de l'Ordovicien du Massif Armoricain et de la Péninsule Ibérique. Senckenbergiana lethaea, 59, 401-429.

Harrison, W. B., III and Harrison, L.K. 1975. A Maquoketalike molluscan community in the Brassfield Formation (Early Silurian) of Adams County, Ohio. Bulletin of American Paleontology, 67 (287), 194-234.

Henry, J.L. 1980. Trilobites ordoviciens du Massif Armoricain. Mémoires Société géologique et minéralogique de Bretagne, 22, 1-250.

Hicks, H. 1873. On the Tremadoc rocks in the neighbourhood of St David's, South Wales and their fossil contents. Quarterly Journal of the Geological Society of London, 29, 39-52.

Mélou, M. 1975. Le genre Heterorthina (Brachiopoda, Orthida) dans la Formation des Schistes de Postolonnec (Ordovicien), Finistère, France. Géobios, 8, 191-208.

Morris, N.J. 1979. On the origin of the Bivalvia. In: The Origin of Major Invertebrate Groups (Ed. M.R. House) Academic Press, London, 381-413.

Munier-Chalmas, M. 1876. Mollusques nouveaux des terrains paléozoïques des environs de Rennes. Journal de Conchyliologie, 3, 102-109.

Newell, N.D. 1965. Classification of the Bivalvia. Novitates, 2206, 1-25.

Péneau, J. 1946. Etude sur l'Ordovicien inférieur (Arénigien $=$ Grès Armoricain) et sa faune (spécialement en Anjou). 
Bulletin de la Société d'Etudes Scientifiques d'Angers, nvelle série, 1944-1946, 37-106.

Pojeta, J., Jr. 1971. Review of Ordovician Pelecypods. Geological Survey Professional Paper, 695, 1-46.

Pojeta, J., Jr. 1978. The origin and early taxonomic diversification of pelecypods. Philosophical Transactions of the Royal Society of London, B 284, 225-246.

Pojeta, J., Jr. 1987. Class Pelecypoda. In: Fossil Invertebrates (Eds. R.S. Boardman, A.H. Cheetham and A.J. Rowell). Blackwell Scientific Publications, 386-435.

Pojeta, J., Jr. and Gilbert-Tomlinson, J., 1977. Australian Ordovician Pelecypod Molluscs. Bureau of Mineral Resources, Geology and Geophysics, Bulletin, 174, 1-64.

Pojeta, J., Jr. and Runnegar, B. 1985. The early evolution of diasome molluscs. In: The Mollusca. Vol. 10, Evolution (Eds. E.R. Trueman and M.R. Clarke). Academic Press, Orlando, 295-336.

Prado, C. de 1855. Mémoire sur la Géologie d'Almadén, d'une partie de la Sierra Morena et des Montagnes de Tolède. Bulletin de la Société géologique de France [2], 12, 182-204.

Prost-Dame, V. 1980. Géologie et gîtologie de la région de Castuera, province de Badajoz (Espagne). Thèse $3 \mathrm{e}$ cycle, Université de Paris-Sud, Centre d'Orsay, 1-288 (inéd.).

Rábano, I. 1989a. Trilobites del Ordovícico Medio del sector meridional de la Zona centroibérica española. Parte I. Yacimientos, bioestratigrafía y aspectos paleobiogeográficos. Boletín Geológico y Minero, $\mathbf{1 0 0}$, 307-338.

Rábano, I. 1989b. Trilobites del Ordovícico Medio del sector meridional de la Zona centroibérica española. Parte III. Calymenina y Cheirurina. Boletín Geológico y Minero, 100, 767-841.

Rábano, I. 1989c. Trilobites del Ordovícico Medio del sector meridional de la Zona centroibérica española. Parte IV. Phacopina, Scutelluina, Odontopleurida y Lichida. Boletín Geológico y Minero, 100, 971-1032.

Rábano, I. 1998. La colección paleontológica de Casiano de Prado conservada en el Museo Geominero (ITGE, Madrid). Geogaceta, 23, 123-125.

Robardet, M. 1981. Evolution géodynamique du Nord-Est du Massif armoricain au Paléozoïque. Mémoires Société géologique et minéralogique de Bretagne, 20, 1-342.

Salter, J.W. 1864. Note on the fossils from the Budleigh Salterton Pebble Bed. Quarterly Journal of the Geological Society of London, 20, 286-302.
San José, M.A., Rábano, I., Herranz, P. y Gutiérrez-Marco, J.C. 1992. Capítulo 28. El Paleozoico inferior de la Zona Centroibérica meridional. In: Paleozoico Inferior de Ibero-América (Eds. J.C. Gutiérrez-Marco, J. Saavedra e I. Rábano). Univ. de Extremadura, 505-521.

Sánchez, T.M. y Suárez-Soruco, R. 1996. Primer registro de lyrodesmatidos (Bivalvia, Heteroconchia) en América del Sur (Formación San Benito, Ordovícico superior de Bolivia). Memorias del XII Congreso geológico de Bolivia, Tarija, 1, 217-219.

Sarmiento, G.N., Gutiérrez-Marco, J.C. and Rábano, I. 1995. A biostratigraphical approach to the Middle Ordovician conodonts from Spain. In: Ordovician Odyssey (Eds. J.D. Cooper, M.L. Droser and S.E. Finney). Pacific Section Society for Sedimentary Geology, Book 77, 6164.

Sharpe, D. 1853. Description of the new species of Zoophyta and Mollusca. In: On the Carboniferous and Silurian formations in the neighbourhood of Bussaco, Portugal (Eds. C. Ribeiro, D. Sharpe and T.R. Jones). Quarterly Journal of the Geological Society of London, 9, 146-158.

Tromelin, G. de 1877. Etude de la faune du grès silurien de May, Jurques, Campandré, Mont-Robert, etc. (Calvados). Bulletin Société linnéenne de Normandie, [3], 1, 5-79.

Tromelin, G. de et Lebesconte, P. 1875. Note sur quelques fossiles des grès siluriens de Saint-Germain/Ille, La Bouëxière, Champeaux, etc. (Ille-et-Vilaine). Ed. Cotonnec, Quimper, 8 pp.

Verneuil, E. de et Barrande, J. 1855. Description des fossiles trouvés dans les terrains silurien et dévonien d'Almadén, d'une partie de la Sierra Morena et des Montagnes de Tolède. Bulletin de la Société géologique de France [2], 12, 964-1025.

Waller, T.R. 1990. The evolution of ligament systems in the Bivalvia. In: The Bivalvia (Ed. B. Morton). Proceedings of a Memorial Symposium in Honour of Sir Charles Maurice Yonge, Edinburgh, 1986. Hong Kong University Press, 49-71.

Wyatt Edgell, A. 1874. Note on some Lamellibranchs of the Budleigh-Salterton pebbles. Quarterly Journal of the Geological Society of London, 30, 45-49.

Young, T.P. 1985. The stratigraphy of the Upper Ordovician of Central Portugal. Ph.D., University of Sheffield, 2 vols., 441 pp. (inéd.).

Ziegler, A.M., Cocks, L.R.M. and Bambach, R.K. 1968. The composition and structure of Lower Silurian marine communities. Lethaia, 1, 1-27. 